\title{
Freqüencia da infecção por Giardia lamblia (Kunstler, 1882) em cães (Canis familiaris) avaliada pelo Método de Faust e cols. (1939) e pela Coloração da Auramina, no município de Canoas, RS, Brasil
}

\author{
Frequence of infection by Giardia lamblia (Kunstler, 1882) in dogs (Canis familiaris) \\ available trough the Faust and Collaborators Method (1939) and Auramine \\ Staining in Canoas, RS, Brazil
}

\section{Cristiane Beck ${ }^{1}$ Flávio Antônio Pacheco de Araújo ${ }^{2}$ Adriana Tarnowski Olicheski ${ }^{3}$ Adriana Schneider Breyer ${ }^{4}$}

\section{RESUMO}

Giardia lamblia é um protozoário que acomete mais comumente animais jovens e que convivem em grupos. Apesar da alta prevalência, nem todos os animais apresentam a forma clínica da doença. Mesmo assim, a giardíase tem importância epidemiológica por possuir um elevado potencial zoonótico. O presente estudo teve como objetivo determinar a freqüência de Giardia lamblia em cães no município de Canoas, RS, Brasil, através do Método de Faust e cols. (1939) e da Técnica de Coloração da Auramina. Os grupos experimentais foram divididos de acordo com a procedência e o sexo. Das 332 amostras analisadas pelo Método de Faust e cols, a freqüencia obtida foi de $34,04 \%$, podendo variar de 28,95 a 39,13\%, dentro de um intervalo de confiança de 95\%. Destas amostras positivas, 40,96\% foram de canil $e$ 27,11\% de cães de rua. O Teste Exato de Fisher aplicado a esses dados revelou existir uma diferença significativa $(p=0,0107)$ entre as variáveis resultado e procedência. A variável sexo, neste método, não apresentou diferença significativa em relação ao resultado $(P=0,8162)$ totalizando $33,11 \%$ de machos $e$ $34,08 \%$ de fêmeas infectadas com o parasita. Das 147 amostras realizadas pela Técnica de Coloração da Auramina, 23 foram positivas, totalizando 15,65\%. A análise estatística pelo Teste McNemar revelou existir diferença significativa entre as duas técnicas ( $P=0,0004)$. O valor Kappa foi igual a 0,07, considerado como um grau de concordância fraco. Os resultados encontrados neste estudo nos permitem afirmar que o Método de Faust e cols. foi o mais adequado para o diagnóstico da infecção por Giardia lamblia, sendo este parasita altamente prevalente na região estudada.

Palavras-chave: Giardia Iamblia, cães, Canoas, método de Faust e colaboradores, técnica de coloração da Auramina, freqüencia, diagnóstico.

\section{ABSTRACT}

Giardia lamblia is a protozoan that commonly infects young animals which live in groups. Despite its high prevalence many animals don't exhibit clinical signs. Giardiasis is epidemiologically important concerning the zoonotic potential of the disease. The objective of this study was to determine the frequence of Giardia Iamblia in the canine population of Canoas, RS, Brazil, applying the Faust and Collaborators Method (1939) and the Auramine Staining Technique. The dogs were classified according to the origin and gender. Feces samples of 332 dogs were analyzed by the Faust and Collaborators Method. The frequency of the infection obtained was $34.04 \%$, ranging from $28.95 \%$ to $39.13 \%$ (confident interval at a 95\% level). For kennel bred animals $40,96 \%$ of the samples were positive and for stray dogs $27.11 \%$. Applying Fisher's Exact Test to these figures

${ }^{1}$ Médico Veterinário, Professor, MSc, Curso de Medicina Veterinária da Universidade de Cruz Alta (UNICRUZ), RS, Rua XV de Novembro, 680, 98700-000, Ijuí, RS. E -mail: beckly@ibest.com.br.

${ }^{2}$ Professor, Médico Veterinário, Doutor, Chefe do Setor de Protozoologia, Faculdade de Veterinária da Universidade Federal do Rio Grande do Sul.

${ }^{3}$ Médico Veterinário Autônomo, MSc.

${ }^{4}$ Graduando do Curso de Medicina Veterinária da Universidade Luterana do Brasil. 
there was a significant difference $(P=0.0107)$ when comparing the origin of the animals and the results of the tests. However when comparing genders there was no significant difference $(P=0.8162)$, the former with $33.11 \%$ of positive males and $34.08 \%$ positive females. When submitted to the Auramine Staining Technique(147 samples), $15.67 \%$ (23) of the samples resulted positive. The statistical analysis using the McNemar Test exposed a significant difference between both techniques $(P=0.0004)$. The Kappa value of 0.07 obtained was considered of low rate. The results allow one to affirm that the Faust and Collaborators Method was the most suitable diagnostic test for Giardia lamblia infection, considering the high prevalence of this parasite in the studied region.

Key words: Giardia lamblia, dogs, Canoas, Faust and collaborators method, Auramina staining, diagnostic, frequence.

\section{INTRODUÇÃO}

Giardia lamblia (também conhecida como Giardia duodenalis ou Giardia intestinalis) é um protozoário entérico que afeta humanos, animais domésticos e silvestres (THOMPSON et al., 2000). Pertencente à ordem Diplomonadida, tem motilidade ativa e se multiplica no intestino, possui um cisto resistente, 0 qual está presente no meio ambiente (OLSON, 2000). De acordo com DÍAZ et al. (1996), o ciclo de vida consiste em dois estágios: trofozoítos e cistos viáveis, os quais contaminam alimentos e água. Os cistos podem ser ingeridos através da água e de alimentos contaminados, mas a transmissão direta também é possível, especialmente em áreas em que os animais se encontram aglomerados como em canis e gatis (LEIB \& ZAJAC, 1999).

Considerar o hospedeiro de origem não constitui um critério válido para a denominação das espécies, uma vez que, embora espécies de Giardia habitem o trato intestinal de todas as classes de vertebrados, a Giardia lamblia é a única espécie identificada encontrada em humanos e na maioria dos mamíferos (SOGAYAR \& GUIMARÃES, 2000).

A infecção por Giardia lamblia pode causar doença clínica moderada a severa, ou permanecer assintomática (OLSON, 2000). A influência de fatores como a cepa do parasita, a duração da infecção, a dieta ou imunidade podem ser importantes para explicar a diversidade de sintomas associados à infecção (BEST CONSULTING,2002).

As maiores prevalências são encontradas nos animais jovens, principalmente até um ano de idade, encontrando-se de 26 a 50\% de animais parasitados, e em canis, o parasita pode ser encontrado em até $100 \%$ dos cães. Por outro lado, em gatos a prevalência é menor, variando de 1,4 a 11\% (ROSEZ et al., 2002).
Nas amostras encaminhadas a laboratórios foi possível observar uma ocorrência de $70 \%$ em Curitiba/PR; 80\% no Rio de Janeiro/RJ; 32\% em Belo Horizonte/MG, e 34\% em Florianópolis/SC. Todos os laboratórios utilizaram o método de flutuação com sulfato de zinco para o diagnóstico (GENNARI \& SOUZA, 2002).

Segundo SHERDING \& JOHNSON (1998), o diagnóstico definitivo depende da identificação dos cistos através da centrifugação - flutuação com sulfato de zinco nas fezes, ou dos trofozoítos flagelados móveis nas fezes diarréicas frescas suspensas em solução salina. TABOADA \& MERCHANT (1997) complementam que devem ser examinadas no mínimo três amostras, devido à produção intermitente de cistos.

Uma técnica pouco conhecida para o diagnóstico de Giardia lamblia, mas utilizado para o diagnóstico de Criptosporidium é a Coloração da Auramina. Segundo DUBEY et al. (1990), é um método simples para a confirmação da presença de oocistos de Cryptosporidium spp. Na literatura, há muitas descrições de coloração para a identificação de oocistos de Cryptosporidium spp, mas muitos laboratórios usam modificações como método de Kinyoun ou auramina, seguido por microscopia fluorescente. Essa técnica foi adaptada para a detecção de cistos de Giardia spp, atualmente utilizada na Espanha (QUADROS, 2002).

Apesar das controvérsias, os autores descrevem que a giardíase deve ser considerada com potencial zoonótico e adequadas precauções devem ser tomadas quando em contato com as fezes ou animais infectados. Com o desenvolvimento da biologia molecular, foi possível a avaliação de diferentes espécies do parasita evidenciando seu potencial zoonótico. Infecções de pessoas são freqüentemente originadas de fontes d'água, ou contato direto com outras pessoas infectadas. Amostras fecais de cães e gatos podem contribuir para a contaminação da água (LEIB \& ZAJAC, 1999).

O presente trabalho foi realizado no município de Canoas, RS, Brasil, visando à determinação da freqüência da infecção por Giardia lamblia nos cães do município, assim como a comparação da sensibilidade do Método de Faust e cols. com a Técnica de Coloração da Auramina para seu diagnóstico.

\section{MATERIAL E MÉTODOS}

A amostragem foi do tipo randômica e estratificada por origem dos animais (canil e rua), de 
acordo com THRUSFIELD (1986), para uma expectativa de prevalência de $30 \%$, com uma precisão absoluta de 5\%, com nível de confiança de 95\%, totalizando 332 amostras.

As amostras de fezes de cães foram coletadas, com ou sem sinal de diarréia. Os animais eram de diferentes raças e idades, provenientes de canis e de rua. Para efeito desse estudo, cães de canis foram considerados aqueles oriundos de propriedades que possuíam no mínimo dois animais, sendo alguns com assistência médico-veterinária. Portanto os cães foram divididos em 2 grupos: grupo 1 - 166 amostras de fezes de cães de canil; grupo 2 - 166 amostras de fezes de cães de rua.

A partir destes grupos, os animais foram classificados em machos e fêmeas. As amostras foram coletadas e analisadas no período de janeiro a julho de 2002. Os proprietários realizavam as coletas logo após defecação espontânea. No caso dos animais de rua, as fezes foram coletadas pela equipe do Canil Municipal, diretamente do reto após a realização da eutanásia.

Após as coletas, as amostras foram acondicionadas em potes plásticos e identificadas. As fezes foram conservadas em geladeira até a realização das análises, até 24 horas após a coleta, no Laboratório de Protozoologia da Universidade Federal do Rio Grande do Sul.

Para o diagnóstico de Giardia Iamblia foram utilizados dois métodos: método de Faust e cols. e a técnica de coloração da Auramina, sendo este último utilizado apenas em 147 amostras. A análise estatística foi realizada através dos seguintes testes.

O teste exato de Fisher ${ }^{4}$ (nível de $\propto=5 \%$ ), foi utilizado nas análises comparativas; o teste de McNemar ${ }^{4}$ foi utilizado nos estudos pareados; a porcentagem de concordância foi calculada para verificar o relacionamento entre as duas técnicas, Faust e cols. e Coloração da Auramina, segundo COUTINHO et al. (1970) e ARAÚJO (1999); a estatística de Kappa, de acordo com SMITH (1995), foi utilizada para medir o grau de concordância real entre as duas técnicas.

\section{RESULTADOS E DISCUSSÃO}

Das amostras analisadas pelo método de Faust e cols., 34,04\% estavam positivas de cães do município de Canoas, resultado semelhante à freqüência encontrada por BARTMANN (2002) em Porto Alegre (37,64\%), 32\% em Belo Horizonte/MG e $34 \%$ em Florianópolis /SC citadas por GENNARI \& SOUZA (2002). No entanto, em Curitiba/PR e Rio de Janeiro/RJ, as freqüências foram superiores, 70 e 80\% respectivamente, analisadas pela mesma técnica (GENNARI \& SOUZA, 2002).

O presente trabalho demonstra que a auramina não é a coloração mais indicada para a realização do diagnóstico de Giardia lamblia, já que somente $15,65 \%$ das amostras examinadas foram positivas, demonstrando ter baixa sensibilidade. Os exames considerados falsos negativos, foram 40, enquanto os falsos positivos foram 13 , resultando em uma diferença significativa entre as duas técnicas e um valor de Kappa igual a 0,07, correspondendo a um grau de concordância fraco.

Para BARR et al. (1992), o diagnóstico de cistos de Giardia spp utilizando o Método de Faust e cols. é ainda considerado o mais prático e efetivo, no entanto, o sucesso do diagnóstico é baixo em cães, particularmente devido à liberação intermitente dos cistos. Portanto há a necessidade de um método mais sensível e específico para o diagnóstico de Giardia lamblia.

BUGG et al. (1999) avaliaram amostras de fezes de cães oriundos de pet shops, abrigos, clínicas veterinárias e canis de criação, de diferentes idades, na Austrália. Foram encontrados 29\% das amostras positivas para Giardia spp em cães refugiados, 37\% em cães de pet shop, e 28,6\% em canis de criação. O resultado das amostras positivas dos cães refugiados se aproxima daquele encontrado neste trabalho, que resultou em $27,11 \%$ de cães de rua parasitados. De forma semelhante ocorreu com os cães de canil, 40,96\% positivos, que, neste trabalho, se assemelham com o grupo de cães de pet shop do autor referido (37\%). A análise das amostras de ambos os trabalhos foi realizada através do Método de Faust e cols.

A alta freqüência de Giardia Iamblia encontrada neste trabalho é preocupante, pois apesar deste protozoário ser de pouca importância clínica em cães, pesquisas têm mostrado que algumas espécies/ genótipos destes parasitas podem ser compartilhados entre humanos e cães particularmente em áreas urbanas (BUGG, 1999). KEULEN et al. (2002) descreve que ainda não está definido se animais agem como reservatórios para humanos ou vice-versa.

ROBERTSON et al. (2000) sugerem que humanos podem ser o principal reservatório para Giardia lamblia e a transmissão direta de pessoa para pessoa é mais importante que a transmissão zoonótica. No entanto, cães e gatos podem carrear espécies de Giardia as quais são potencialmente infectivas para seres humanos, e que o potencial zoonótico deve ser considerado, especialmente para pessoas imunocomprometidas. CIMERMAN et al. (1999) 
relatam uma prevalência de $16 \%$ em pacientes aidéticos.

Apesar de não ocorrer diferença significativa na infecção entre machos e fêmeas para o protozoário, OLIVEIRA-SEQUEIRA \& AMARANTE (2001), encontraram maior número de machos adultos positivos do que fêmeas. Os autores acrescentam que cães castrados tendem a mostrar uma reduzida prevalência da infecção comparando-se com animais sexualmente ativos. Por outro lado, HOREJS \& KOUDELA (1994) observaram um resultado diferente, em que houve maior freqüência de Giardia spp em fêmeas do que em machos, tendo como resultados $7 \%$ e 3,4\%, respectivamente. As diferenças encontradas nos diversos trabalhos da literatura podem estar relacionados a uma não padronização do método principalmente ao que se refere à densidade da solução flutuadora, bem como a diferentes condições de criação e manejo que afetariam a taxa de exposição ao risco dos animais.

\section{CONCLUSÃO}

Após realizar as análises dos resultados obtidos neste estudo, concluiu-se que a freqüência da infecção por Giardia Iamblia em cães em Canoas, RS, é expressiva. Neste trabalho observouse que o método de Faust e cols., é o mais adequado para o diagnóstico de Giardia Iamblia do que a técnica de coloração da Auramina e que cães de canil apresentam maiores freqüências de infecção por Giardia lamblia do que cães de rua.

\section{REFERÊNCIAS BIBLIOGRÁFICAS}

ARAÚJO, F.A.P. Avaliação soroepidemiológica de anticorpos para Toxoplasma gondii Nicolle \& Manceaux, 1909 em soros de suínos (Sus scrofa) da Região da Grande Erechim, RSBrasil, detectados através das Técnicas de imunofluorescência indireta de imunoenzimática. 1999. 125 f. Tese (Doutorado em Parasitologia) - Instituto Oswaldo Cruz.

BARR, S.C. et al. Evaluation of two test procedures for diagnosis of giardiasis in dogs. American Journal Veterinary Research, v.53, p.2028-2031, 1992.

BARTMAN, A. Freqüência de Giardia lamblia (Kunstler, 1882), em cães (Canis familiaris) determinada através de exames parasitológicos solicitados por clínicas veterinárias da cidade de Porto Alegre, Rio Grande do Sul, Brasil. 2002. $80 \mathrm{f}$. Dissertação (Mestrado em Parasitologia) - Universidade Federal do Rio Grande do Sul.

BEST CONSULTING. Giardia. Disponível em:<http:// www.bestconsulting.com/veterinaria/giardia/giardia.htm>. On line. Acesso em 22 abr. 2002.

BUGG, R.J. et al. Gastrointestinal parasites of urban dogs in Perth, Western Austrália. Veterinary Journal, v.157, p.295301, 1999.

CIMERMAN, S. et al. Prevalence of intestinal parasitic infections in patients with acquired immunodeficiency syndrome in Brazil. International Journal of Infections Diseases, v.3, p.203-206, 1999.

COUTINHO, S.G. et al. Análise comparativa entre as sensibilidades da reação indireta de anticorpos fluorescentes e da reação de Sabin-Feldman na pesquisa de anticorpos séricos para toxoplasmose. Revista da Sociedade Brasileira de Medicina Tropical, Rio de Janeiro, v.IV, n.5, p.315-325, 1970.

DÍAZ, V. et al. Aspects of animal giardioses in Granada province (Southern Spain). Veterinary Parasitology, v.64. p.171-176, 1996.

DUBEY, J.P. et al. Cryptosporidiosis of man and animals. CRC, 1990. 199p.

GENNARI, S.M.; SOUZA, S. Giardíase. São Paulo : Fort Dodge Saúde Animal LTDA, 2002. 13p. (Boletim Técnico).

HOREJS, R.; KOUDELA, B. Giardiasis in dogs in a breeding kennel. Veterinary Medicine, v.39, p.93-101, 1994.

KEULEN, H. et al. Presence of human Giardia in domestic, farm and wild animals, and environmental samples suggests a zoonotic potential for giardiasis. Veterinary Parasitology, v.108, p.97-107, 2002.

LEIB, M.S.; ZAJAC, A.M. Giardiasis in dogs and cats. Veterinary Medicine, v.94, n.9, p.793-802, 1999.

OLIVEIRA-SEQUEIRA, T.C.G.; AMARANTE, A.F.T. Prevalence of intestinal parasites in dogs from São Paulo State, Brazil. Veterinary Parasitology, v.103, p.19-27, 2001.

OLSON, M.E. A Giardíase e o uso da vacinação para o controle da infecção. [S.l.: s.n], 2000.

QUADROS, R.M. Ocorrência de Cryptosporidium spp (Tyzzer, 1907) detectada pelo método de imunofluorescência através da técnica de coloração da auramina em propriedades rurais do município de Lages (SC), Brasil. 2002. 66f. Dissertação (Mestrado em Parasitologia) - Universidade Federal do Rio Grande do Sul, 2002.

ROBERTSON, I.D. et al. The role of companion animals in the emergence of parasitic zoonoses. International Journal for Parasitology, v.30, p.1369-1377, 2000.

ROSEZ, K.V. et al. Giardia: uma infecção global. Revista Nosso Clínico, n.26, p.30-34, 2002.

SMITH, R.D. Veterinary clinical epidemiology, a problem-oriented appoach. 2.ed. CRC, 1995. 279p.

SHERDING, R.G.; JOHNSON, S.E. Doenças dos intestinos. In: BIRCHARD, S.J.; SHERDING, R.G. Manual Saunders: clínica de pequenos animais. São Paulo : Roca, 1998. p.771-803. 
SOGAYAR, M.I.T.L.; GUIMARÃES, S. Giardia lamblia. In: NEVES, D.P. Parasitologia humana. 10.ed. São Paulo : Atheneu, 2000. Cap.14, p.107-113.

TABOADA, J.; MERCHANT, S.R. Infecções por protozoários e por outras causas. In: ETTINGER, S.J.; FELDMAN E.C. Tratado de medicina interna veterinária. 4.ed. São Paulo: Manole, 1997. V.1, cap.68, p.554-572.
THOMPSON, A.R.C. et al. Nomenclature and genetic groupings of Giardia infecting mammals. Parasitology Today, v.16, n.5, p.210-217, 2000.

THRUSFIELD, M. Veterinary epidemiology. Great Britain: Butterworth, 1986. 483p. 